\title{
THE ZEROES OF NONNEGATIVE CURVATURE OPERATORS
}

\author{
JOHN A. THORPE
}

The Riemannian sectional curvature of a Riemannian manifold is a realvalued function $\sigma$ on the Grassmann bundle of tangent 2-planes of $M$. Although there exists a large body of theorems relating the curvature of $M$ to various topological and geometric properties of $M$, relatively little is known of a general nature about the behavior of the function $\sigma$ itself. In particular, the critical point behavior of $\sigma$ has been analyzed only in very special cases [3], [4]. In this paper we consider the pointwise behavior of $\sigma$; that is, we consider the restriction of $\sigma$ to the Grassmann manifold of tangent 2-planes at a point $m \in M$. We are then able to describe completely the structure of the sets of points in this manifold where $\sigma$ assumes its minimum and maximum. In particular, for spaces of nonnegative curvature we describe the set of points where $\sigma$ assumes the value zero.

To be more specific, let $G$ denote the Grassmann manifold of oriented tangent 2-planes at $m$. $G$ is in a natural way a submanifold of the vector space $\Lambda^{2}$ of 2 -vectors at $m$. Since $G$ is a 2 -fold covering space of the manifold of (unoriented) 2-planes at $m$, we may regard $\sigma$ as a function on $G$. We shall show that the minimum and maximum sets of $\sigma$ are intersections with $G$ of linear subspaces of $\Lambda^{2}$. Moreover every such intersection can occur, for example as the minimum set of some curvature function $\sigma$ on $G$.

The case of nonnegative curvature $\sigma \geq 0$ will occupy most of our attention here. One reason for this is that the general result on the minimum set of $\sigma$ is an elementary consequence of the result for $\sigma \geq 0$, and another is that this case is the one most likely to yield applications. For example, it follows from our description of the minimum set that if $\sigma \geq 0$ and relative to some coordinate system the "diagonal" curvature components $R_{i j i j}$ are all zero at $m$, then in fact the curvature tensor $R$ is zero at $m$.

Given a space $M$ of nonnegative curvature and given $m \in M$, the linear subspace of $\Lambda^{2}$ whose intersection with $G$ is the zero set of $\sigma$ is obtained as follows. The curvature tensor $R$ of $M$ at $m$ can be regarded as a self-adjoint linear operator on $\Lambda^{2}$. Letting $\mathscr{R}$ denote the vector space of all self-adjoint linear operators ("curvature operators") on $\Lambda^{2}$, the subset $\mathscr{B}$ consisting of those

Communicated by I. M. Singer, December 17, 1969. Research partially supported by the National Science Foundation. 
which come from Riemannian structures (i.e., those satisfying the first Bianchi identity) is a linear subspace of $\mathscr{R}$. The orthogonal complement $\mathscr{S}$ of $\mathscr{B}$ in $\mathscr{R}$ is the set of all curvature operators whose associated Riemannian curvature function is identically zero. Our theorem asserts that there exists an operator $S \in \mathscr{S}$ such that the zero set of $\sigma$ (also called the zero set of $R$ ) is precisely $G \cap \operatorname{Ker}(R-S)$.

The idea of the proof is first to show that for each $P$ in the zero set there exists an $S \in \mathscr{S}$ such that $P \in \operatorname{Ker}(R-S)$, second to observe that there is a unique such $S$ orthogonal to the subspace of $\mathscr{S}$ annihilating $P$, and finally to piece these unique operators together to build one which works simultaneously for all $P$ in the zero set.

The author wishes to thank J. Simons for several stimulating discussions of the ideas presented here.

\section{1. $\mathscr{S}$ and the Grassmann quadratic 2-relations}

We begin by analyzing the space $\mathscr{S}$ complementary in $\mathscr{R}$ to the subspace $\{R \in \mathscr{R} \mid R$ satisfies the Bianchi identity $\}$. We shall exhibit a natural isomorphism between $\mathscr{S}$ and $\Lambda^{4}$ and shall establish the relationship between $\mathscr{S}$ and the Grassmann quadratic 2-relations which are necessary and sufficient conditions for decomposability of elements in $\Lambda^{2}$.

Let $V$ be an $n$-dimensional real vector space with inner product $\langle$,$\rangle (e.g.,$ $V=$ the tangent space at a point of a Riemannian manifold). For $k$ an integer $\geq 0$, let $\Lambda^{k}=\Lambda^{k}(V)$ denote the space of $k$-vectors of $V$, equipped with inner product given by

$$
\left\langle u_{1} \wedge \cdots \wedge u_{k}, v_{1} \wedge \cdots \wedge v_{k}\right\rangle=\operatorname{det}\left[\left\langle u_{i}, v_{j}\right\rangle\right], \quad u_{i}, v_{i} \in V .
$$

Let $G$ denote the Grassmann manifold of oriented 2-dimensional subspaces of $V$; we identify $G$ with the submanifold of $\Lambda^{2}$ consisting of decomposable 2-vectors of length 1 by $P \leftrightarrow u \wedge v$ where $\{u, v\}$ is any oriented orthonormal basis for $P$. Let $\mathscr{R}$ denote the space of self-adjoint linear operators on $\Lambda^{2}$, equipped with inner product given by $\langle R, S\rangle=\operatorname{trace} R \circ S, R, S \in \mathscr{R}$. Elements of $\mathscr{R}$ will be called curvature operators on $V$. Given $R \in \mathscr{R}$, its sectional curvature is the function $\sigma_{R}: G \rightarrow R$ defined by $\sigma_{R}(P)=\langle R P, P\rangle, P \in G$. Each $R \in \mathscr{R}$ can be naturally identified with a 2 -form on $V$ with values in the vector space of skew-symmetric endomorphisms of $V$ by

$$
\langle R(u, v)(w), x\rangle=R(u \wedge v, w \wedge x), \quad u, v, w, x \in V .
$$

We can then consider the subspace $\mathscr{B}$ of $\mathscr{R}$ consisting of those $R \in \mathscr{R}$ which satisfy the first Bianchi identity: $R \in \mathscr{B}$ if and only if

$$
R(u, v) w+R(v, w) u+R(w, u) v=0
$$


for all $u, v, w \in V$. Set $\mathscr{S}=\mathscr{B}^{\perp}$, the orthogonal complement of $\mathscr{B}$ in $\mathscr{R}$.

We construct, for each $\xi \in \Lambda^{4}$, an operator $S_{\xi} \in \mathscr{S}$ as follows. Given $\xi$, define $S_{\xi}: \Lambda^{2} \rightarrow \Lambda^{2}$ by

$$
\left\langle S_{\xi}(\alpha), \beta\right\rangle=\langle\alpha \wedge \beta, \xi\rangle, \quad \alpha, \beta \in \Lambda^{2} .
$$

Clearly $S_{\xi} \in \mathscr{R}$. To see that $S_{\xi} \in \mathscr{S}$ we need the following

Lemma 1.1. Let $\left\{e_{1}, \cdots, e_{n}\right\}$ be an orthonormal basis for $V$. For $1 \leq$ $i, j, k, l \leq n$, set $S_{i j k l}=S_{e_{i} \wedge e_{j} \wedge e_{k} \wedge e_{l}}$. Then, for $R \in \mathscr{R}$,

$$
\begin{aligned}
\left\langle R, S_{i j k l}\right\rangle=2\left[\left\langle R\left(e_{i} \wedge e_{j}\right), e_{k} \wedge e_{l}\right\rangle\right. & +\left\langle R\left(e_{j} \wedge e_{k}\right), e_{i} \wedge e_{l}\right\rangle \\
& \left.+\left\langle R\left(e_{k} \wedge e_{i}\right), e_{j} \wedge e_{l}\right\rangle\right] .
\end{aligned}
$$

Proof.

$$
\begin{aligned}
& \left\langle R, S_{i j k l}\right\rangle=\operatorname{tr} R \circ S_{i j k l}=\sum_{\alpha<\beta}\left\langle R \circ S_{i j k l}\left(e_{\alpha} \wedge e_{\beta}\right), e_{\alpha} \wedge e_{\beta}\right\rangle \\
& =\sum_{\alpha<\beta}\left\langle S_{i j k l}\left(e_{\alpha} \wedge e_{\beta}\right), R\left(e_{\alpha} \wedge e_{\beta}\right)\right\rangle \\
& =\sum_{\alpha<\beta}\left\langle S_{i j k l}\left(e_{\alpha} \wedge e_{\beta}\right), \sum_{\gamma<\delta}\left\langle R\left(e_{\alpha} \wedge e_{\beta}\right), e_{\gamma} \wedge e_{\delta}\right\rangle e_{\gamma} \wedge e_{\delta}\right\rangle \\
& =\sum_{\alpha<\beta} \sum_{\gamma<\delta}\left\langle R\left(e_{\alpha} \wedge e_{\beta}\right), e_{\gamma} \wedge e_{\dot{\delta}}\right\rangle \\
& \times\left\langle e_{\alpha} \wedge e_{\beta} \wedge e_{r} \wedge e_{\delta}, e_{i} \wedge e_{j} \wedge e_{k} \wedge e_{l}\right\rangle .
\end{aligned}
$$

Collecting terms completes the proof.

Proposition 1.2. $\xi \mapsto S_{\xi}$ maps $\Lambda^{4}$ isomorphically onto $\mathscr{S}$. Moreover $\xi \mapsto$ $(1 / \sqrt{6}) S_{\xi}$ is an isometry.

Proof. Clearly $\xi \mapsto S_{\xi}$ is a linear map from $\Lambda^{4}$ into $\mathscr{R}$. Since $\left\{e_{i} \wedge e_{j} \wedge\right.$ $\left.e_{k} \wedge e_{l} \mid i<j<k<l\right\}$ is an (orthonormal) basis for $\Lambda^{4}$, and the images $S_{i j k l}$ of the basis vectors are all in $\mathscr{S}\left(\left\langle R, S_{i j k l}\right\rangle=0\right.$ for all $R \in \mathscr{B}$ by Lemma 1.1), it follows that $\xi \mapsto S_{\xi}$ maps $\Lambda^{4}$ into $\mathscr{S}$. In fact, Lemma 1.1 implies that, given $R \in \mathscr{R}, R \in \mathscr{B}$ if and only if $\left\langle R, S_{i j k l}\right\rangle=0$ for all $i, j, k, l$; i.e., the $S_{i j k l}$ span $\mathscr{S}$ and $\xi \mapsto S_{\xi}$ maps onto $\mathscr{S}$. Injectivity and the fact that $\xi \mapsto(1 / \sqrt{6}) S_{\xi}$ is an isometry follow from taking $R=S_{\alpha \beta \gamma \delta}$ in Lemma 1.1 to conclude that $\left\{S_{i j k l} \mid i<j<k<l\right\}$ is an orthogonal set and that $\left\|S_{i j k l}\right\|^{2}=6$.

Remark. Using the natural isomorphism between $\Lambda^{4}$ and its dual, the space of alternating 4-forms on $V$, given by the inner product we can also identify $\mathscr{S}$ with this space of 4-forms. Explicitly, one identifies a 4-form $\omega$ on $V$ with the operator $S_{\omega} \in \mathscr{S}$ given by

$$
\left\langle S_{\omega}\left(v_{1} \wedge v_{2}\right), v_{3} \wedge v_{4}\right\rangle=\omega\left(v_{1}, v_{2}, v_{3}, v_{4}\right)
$$

Proposition 1.3. Let $\alpha \in \Lambda^{2}$. Then $\alpha$ is decomposable if and only if $\langle S \alpha, \alpha\rangle$ $=0$ for all $S \in \mathscr{S}$.

Proof. The necessity of the condition is clear since each $S \in \mathscr{S}$ is of the form $S_{\xi}$ for some $\xi \in \Lambda^{4}$ and $\left\langle S_{\xi} \alpha, \alpha\right\rangle=\langle\alpha \wedge \alpha, \xi\rangle=0$ for $\alpha$ decomposable. 
Conversely. given an orthonormal basis $\left\{e_{1}, \cdots, e_{n}\right\}$ for $V$, it is well-known [2, p. $309 \mathrm{ff}$ ] (see also [1]) that the conditions $\left\langle S_{i j k l} \alpha, \alpha\right\rangle=0$ for all $i<j<k<l$ are necessary and sufficient conditions for decomposability.

Remark. The conditions $\left\langle S_{i j k l} \alpha, \alpha\right\rangle=0$ are known as the Grassmann quadratic 2-relations.

Remark. It is clear from Proposition 1.3 that each curvature tensor $S \in \mathscr{S}$ has sectional curvature $\sigma_{S}$ identically zero. Conversely, it is easily checked that this property characterizes $\mathscr{S}$.

\section{The uniqueness theorem}

In this section we establish the basic uniqueness result which is at the heart of our building process. But first we need some additional notation.

For a subset $Z$ of $G$, let

$$
\mathscr{A}(Z)=\{S \in \mathscr{S} \mid S(P)=0 \text { for all } P \in Z\} .
$$

Thus $\mathscr{A}(Z)$ is the subspace of $\mathscr{S}$ consisting of all elements of $\mathscr{S}$ which annihilate $Z$. For a finite subset $Z=\left\{P_{1}, \cdots, P_{k}\right\}$ of $G$, we shall denote $\mathscr{A}\left(\left\{P_{1}, \cdots, P_{k}\right\}\right)$ simply by $\mathscr{A}\left(P_{1}, \cdots, P_{k}\right)$. By $\mathscr{A}(Z)^{\perp}$ with $Z \subset G$ we shall mean the orthogonal complement of $\mathscr{A}(Z)$ in $\mathscr{S}$.

Theorem 2.1. Let $R \in \mathscr{R}$ and $Z \subset G$, and suppose there exists $S \in \mathscr{S}$ such that $Z \subset \operatorname{Ker}(R-S)$. Then there exists a unique $S_{0} \in \mathscr{A}(Z)^{\perp}$ such that $Z \subset \operatorname{Ker}\left(R-S_{0}\right)$. Moreover, given any $S \in \mathscr{S}, Z \subset \operatorname{Ker}(R-S)$ if and only if the orthogonal projection of $S$ onto $\mathscr{A}(Z)^{\perp}$ is $S_{0}$.

Proof. Existence: Let $S \in \mathscr{S}$ be such that $Z \subset \operatorname{Ker}(R-S)$, and let $S_{0}$ denote the orthogonal projection of $S$ onto $\mathscr{A}(Z)^{\perp}$. Then $S=S_{0}+S^{\prime}$ for some $S^{\prime} \in \mathscr{A}(Z)$ and

$$
Z \subset \operatorname{Ker}(R-S) \cap \operatorname{Ker} S^{\prime} \subset \operatorname{Ker}\left(R-S+S^{\prime}\right)=\operatorname{Ker}\left(R-S_{0}\right) .
$$
Then

Uniqueness: Suppose $Z \subset \operatorname{Ker}\left(R-S_{0}\right) \cap \operatorname{Ker}\left(R-S_{0}^{\prime}\right)$ for $S_{0}, S_{0}^{\prime} \in \mathscr{A}(Z)^{\perp}$.

$$
Z \subset \operatorname{Ker}\left[\left(R-S_{0}\right)-\left(R-S_{0}^{\prime}\right)\right]=\operatorname{Ker}\left(S_{0}^{\prime}-S_{0}\right) .
$$

Thus $S_{0}^{\prime}-S_{0} \in \mathscr{A}(Z)$. But $S_{0}^{\prime}$ and $S_{0} \in \mathscr{A}(Z)^{\perp}$, so $S_{0}^{\prime}-S_{0}$ must be zero.

Finally, it is immediate from the above existence and uniqueness arguments that $Z \subset \operatorname{Ker}(R-S)$ implies $S_{0}$ is the orthogonal projection of $S$ onto $\mathscr{A}(Z)^{\perp}$. Conversely, if $S \in \mathscr{S}$ is such that its orthogonal projection onto $\mathscr{A}(Z)^{\perp}$ is $S_{0}$, then $S=S_{0}+S^{\prime}$ for some $S^{\prime} \in \mathscr{A}(Z)$ and

$$
Z \subset \operatorname{Ker}\left(R-S_{0}\right) \cap \operatorname{Ker} S^{\prime} \subset \operatorname{Ker}\left(R-S_{0}-S^{\prime}\right)=\operatorname{Ker}(R-S) .
$$

Remark. Note that if $R \in \mathscr{R}, S \in \mathscr{S}$ and $P \in G \cap \operatorname{Ker}(R-S)$, then 


$$
\sigma_{R}(P)=\langle R P, P\rangle=\langle S P, P\rangle=\sigma_{S}(P)=0 .
$$

In particular, setting

$$
Z(R)=\left\{P \in G \mid \sigma_{R}(P)=0\right\},
$$

we see that if, for some $S \in \mathscr{S}$, the subspace $\operatorname{Ker}(R-S)$ has non-null intersection with $G$ then the set $Z(R)$ of zeroes of $\sigma_{R}$ is at least big enough to contain this intersection.

Theorem 2.2. Let $R \in \mathscr{R}$, and suppose there exists $S \in \mathscr{S}$ such that $Z(R)$ $=G \cap \operatorname{Ker}(R-S)$. Then there exists a unique $S_{0} \in \mathscr{A}(Z(R))^{\perp}$ such that $Z(R)=G \cap \operatorname{Ker}\left(R-S_{0}\right)$.

Proof. By Theorem 2.1, there exists a unique $S_{0} \in \mathscr{A}(Z(R))^{\perp}$ such that $Z(R) \subset G \cap \operatorname{Ker}\left(R-S_{0}\right)$. But, by the remark above, $G \cap \operatorname{Ker}\left(R-S_{0}\right)$ $\subset Z(R)$. Hence we have the equality.

\section{Critical zeroes}

In studying the critical points of curvature functions, it suffices to consider critical points with critical value zero. For if $\lambda$ is a critical value of $\sigma_{R}, R \in \mathscr{R}$, then the set of critical points of $\sigma_{R}$ with critical value $\lambda$ is the same as the set of critical points of $\sigma_{R-\lambda I}$ with critical value zero, $I$ being the identity operator on $\Lambda^{2}$. In this section we show that if $P$ is a critical zero of $\sigma_{R}$, then $P \in \operatorname{Ker}(R-S)$ for some $S \in \mathscr{S}$.

Lemma 3.1. Let $P \in G$, and let $\left\{e_{1}, \cdots, e_{n}\right\}$ be an orthonormal basis for V. Then

$$
\{P\} \cup\left\{S_{i j k l}(P) \mid i<j<k<l\right\}
$$

spans the normal space to $G \subset \Lambda^{2}$ at $P$. If the basis is chosen so that $P=e_{1} \wedge e_{2}$, then

$$
\{P\} \cup\left\{S_{12 k l}(P) \mid 2<k<l\right\}
$$

is an orthonormal basis for this normal space.

Proof. By Proposition 1.3,

$$
G=\left\{\alpha \in \Lambda^{2} \mid\langle\alpha, \alpha\rangle=1 \text { and }\left\langle S_{i j k l}(\alpha), \alpha\right\rangle=0 \text { for all } i<j<k<l\right\} \text {. }
$$

Since the real valued functions $\alpha \mapsto\langle\alpha, \alpha\rangle$ and $\alpha \mapsto\left\langle S_{i j k l} \alpha, \alpha\right\rangle$ are constant on $G$, their gradients $2 P$ and $2 S_{i j k l}(P)$ at $P \in G$ must be normal to $G$ at $P$. To see that they span the normal space $N_{P}$ of $G$ at $P$, consider first the case where $P=e_{1} \wedge e_{2}$. Then, for $i<j<k<l$,

$$
S_{i j k l}(P)= \begin{cases}e_{k} \wedge e_{l}, & \text { for }(i, j)=(1,2), \\ 0, & \text { for }(i, j) \neq(1,2) .\end{cases}
$$

It follows that, in this case, $\{P\} \cup\left\{S_{12 k l}(P) \mid 2<k<l\right\}$ is an orthonormal set 
in $N_{P}$. Now the number $[(n-2)(n-3) / 2]+1$ of elements in this set is equal to the codimension $[n(n-1) / 2]-2(n-2)$ of $G$ in $\Lambda^{2}$ which in turn is equal to the dimension of $N_{P}$. Hence $\{P\} \cup\left\{S_{12 k l}(P) \mid 2<k<l\right\}$ is an orthonormal basis for $N_{P}$.

Returning to the general case, let $\left\{e_{1}, \cdots, e_{n}\right\}$ be an arbitrary orthonormal basis for $V$, and let $\left\{e_{1}^{\prime}, \cdots, e_{n}^{\prime}\right\}$ be one such that $P=e_{1}^{\prime} \wedge e_{2}^{\prime}$. Let $\left\{S_{i j k l} \mid\right.$ $i<j<k<l\}$ and $\left\{S_{i j k l}^{\prime} \mid i<j<k<l\right\}$ be the corresponding bases for $\mathscr{S}$. Then, from above, $\{P\} \cup\left\{S_{12 k l}^{\prime}(P) \mid 2<k<l\right\}$ spans $N_{P}$. But each $S_{12 k l}^{\prime}$ is a linear combination of the $S_{i j k l}$ and hence each $S_{12 k l}^{\prime}(P)$ is a linear combination of the $S_{i j k l}(P)$. Thus $\{P\} \cup\left\{S_{i j k l}(P) \mid i<j<k<l\right\}$ spans $N_{P}$.

Theorem 3.2. Let $R \in \mathscr{R}$ and suppose $P \in G$ is a critical zero of $\sigma_{R}$. Then there exists $S \in \mathscr{S}$ such that $P \in \operatorname{Ker}(R-S)$.

Proof. Let $\left\{e_{1}, \cdots, e_{n}\right\}$ be an orthonormal basis for $V$ such that $P=e_{1} \wedge e_{2}$. Since $P$ is a critical point of $\sigma_{R}$, and $\sigma_{R}$ is the restriction to $G$ of the function $\alpha \mapsto\langle R(\alpha), \alpha\rangle$, the gradient $2 R(P)$ of this function at $P$ must be normal to $G$ at $P$. By Lemma 3.1, this implies that

$$
R P=\lambda P+\sum_{2<k<l} \mu_{k l} S_{12 k l}(P)
$$

for some $\lambda, \mu_{k l} \in R$. But $\lambda=\langle R P, P\rangle=\sigma_{R}(P)=0$, so $P \in \operatorname{Ker}(R-S)$ where $S=\sum_{2<k<l} \mu_{k l} S_{12 k l}$.

Corollary 3.3. Let $R \in \mathscr{R}$ and suppose $P \in G$ is a critical zero of $\sigma_{R}$. Then there exists a unique $S \in \mathscr{A}(P)^{\perp}$ such that $P \in \operatorname{Ker}(R-S)$.

Proof. Immediate from Theorems 3.2 and 2.1.

Remark. The operator $S$ constructed in the proof of Theorem 3.2 is in fact the unique $S \in \mathscr{A}(P)^{\perp}$ such that $P \in \operatorname{Ker}(R-S)$. Indeed, by Lemma 1.1 together with the fact that each $S^{\prime} \in \mathscr{S}$ is an $S_{\omega}$ for some alternating 4-form $\omega$ on $V$, we have

$$
\left\langle S^{\prime}, S_{12 k l}\right\rangle=6\left\langle S^{\prime}\left(e_{1} \wedge e_{2}\right), e_{k} \wedge e_{l}\right\rangle,
$$

and this is zero for all $S^{\prime} \in \mathscr{A}(P)$; thus $S_{12 k l} \in \mathscr{A}(P)^{\perp}$ for $2<k<l$.

Note also that, since $\left\{S_{12 k l} \mid 2<k<l\right\}$ is linearly independent, the numbers $\mu_{k l}$ above are uniquely determined. In fact, they are curvature components of $R$ relative to the basis $\left\{e_{i}\right\}$ :

$$
\begin{aligned}
\mu_{k l} & =\left\langle\sum_{2<\alpha<\beta} \mu_{\alpha \beta} e_{\alpha} \wedge e_{\beta}, e_{k} \wedge e_{l}\right\rangle=\left\langle\sum_{2<\alpha<\beta} \mu_{\alpha \beta} S_{12 \alpha \beta}\left(e_{1} \wedge e_{2}\right), e_{k} \wedge e_{l}\right\rangle \\
& =\left\langle R\left(e_{1} \wedge e_{2}\right), e_{k} \wedge e_{l}\right\rangle .
\end{aligned}
$$

\section{The case $n=4$}

We consider now the case when $V$ has dimension 4, and establish our main theorem in this case. The validity of the result in dimension 4 will play a crucial role in establishing the theorem in general. 
Theorem 4.1. Let $\operatorname{dim} V=4$, and suppose $R \in \mathscr{R}$ is such that $\sigma_{R} \geq 0$ and $Z(R) \neq \emptyset$. Then there exists a unique $S \in \mathscr{S}$ such that $Z(R)=G \cap \operatorname{Ker}(R-S)$.

Proof. Since $\operatorname{dim} V=4, \mathscr{S}$ is 1 -dimensional. Given $\left\{e_{1}, \cdots, e_{4}\right\}$ an orthonormal basis for $V$, the operator $S_{1234}$ is just the Hodge star operator $*$ and so $\{*\}=\left\{S_{1234}\right\}$ is a basis for $\mathscr{S}$. Given $P \in Z(R), P$ is a minimum, hence a critical point, of $\sigma_{R}$ so by Theorem 3.2 there exists $\mu \in R$ such that $P \in \operatorname{Ker}(R-\mu *)$; i.e., such that

$$
R P=\mu * P .
$$

If $P_{1}$ and $P_{2}$ are two zeroes of $\sigma_{R}$, then $R P_{i}=\mu_{i} * P_{i}$ for some $\mu_{i} \in R(i=1,2)$. We shall show that $\mu_{1}=\mu_{2}$. This is clear if $\left\{P_{1}, P_{2}\right\}$ is linearly dependent in $\Lambda^{2}$, so we may assume linear independence. We have

$$
\mu_{1}\left\langle * P_{1}, P_{2}\right\rangle=\left\langle R P_{1}, P_{2}\right\rangle=\left\langle P_{1}, R P_{2}\right\rangle=\mu_{2}\left\langle P_{1}, * P_{2}\right\rangle=\mu_{2}\left\langle * P_{1}, P_{2}\right\rangle .
$$

Hence, if $\left\langle * P_{1}, P_{2}\right\rangle \neq 0$ we must have $\mu_{1}=\mu_{2}$. On the other hand, if $\left\langle * P_{1}, P_{2}\right\rangle$ $=0$, then $\left\langle P_{1}+P_{2}, *\left(P_{1}+P_{2}\right)\right\rangle=0$, so $P_{1}+P_{2}$ is decomposable. Let $Q=\left(P_{1}+P_{2}\right) / l$ where $l=\left\|P_{1}+P_{2}\right\|$. Then $Q \in G$ and

$$
R Q=\left(\mu_{1} * P_{1}+\mu_{2} * P_{2}\right) / l,
$$

so $\sigma_{R}(Q)=\langle R Q, Q\rangle=0$. Thus $Q$ is also a zero of $\sigma_{R}$; hence $R Q=\mu * Q$ for some $\mu \in R$, and

$$
\mu_{1} * P_{1}+\mu_{2} * P_{2}=l R Q=l \mu * Q=\mu\left(* P_{1}+* P_{2}\right) .
$$

This implies that

$$
\left(\mu_{1}-\mu\right) P_{1}+\left(\mu_{2}-\mu\right) P_{2}=0 \text {, }
$$

and hence $\mu_{1}=\mu_{2}=\mu$ since $\left\{P_{1}, P_{2}\right\}$ is linearly independent in $\Lambda^{2}$.

It follows that $Z(R) \subset \operatorname{Ker}(R-\mu *)$ for some unique $\mu \in \boldsymbol{R}$. By the Remark in $\S 2, G \cap \operatorname{Ker}(R-\mu *) \subset Z(R)$. Hence, setting $S=\mu *$ we have $Z(R)$ $=G \cap \operatorname{Ker}(R-S)$.

Corollary 4.2. Let $\operatorname{dim} V=4$ and $R \in \mathscr{R}$, and let $\lambda$ denote the minimum (or maximum) value of $\sigma_{R}$. Then there exists a unique $S \in \mathscr{S}$ such that

$$
\left\{P \in G \mid \sigma_{R}(P)=\lambda\right\}=G \cap \operatorname{Ker}(R-\lambda I-S) .
$$

Proof. Follows immediately from Theorem 4.1 upon replacing $R$ in that theorem by $R-\lambda I$ (or, in the case where $\lambda$ is the maximum value of $\sigma_{R}$, by $\lambda I-R)$.

Remark. The hypotheses of Corollary 4.2 cannot by weakened to include the case where $\lambda$ is an arbitrary critical value of $\sigma_{R}$. Indeed, if we define $R \in \mathscr{R}$ by 


$$
\begin{array}{ll}
R\left(e_{1} \wedge e_{2}\right)=e_{3} \wedge e_{4}, & R\left(e_{3} \wedge e_{4}\right)=e_{1} \wedge e_{2}, \\
R\left(e_{1} \wedge e_{3}\right)=0, & R\left(e_{2} \wedge e_{4}\right)=0, \\
R\left(e_{2} \wedge e_{3}\right)=-e_{1} \wedge e_{4}, & R\left(e_{1} \wedge e_{4}\right)=-e_{2} \wedge e_{3},
\end{array}
$$

then each of the basis planes $e_{i} \wedge e_{j}$ is a critical zero of $\sigma_{R}$ (critical because $\left(\operatorname{grad} \sigma_{R}\right)\left(e_{i} \wedge e_{j}\right)=2 R\left(e_{i} \wedge e_{j}\right)= \pm 2 * e_{i} \wedge e_{j}$ which is normal to $G$ at $\left.e_{i} \wedge e_{j}\right)$. Hence, if either $\sigma_{R}^{-1}(0)$ or the critical set of $\sigma_{R}$ with critical value zero were the intersection of $G$ with a linear subspace of $\Lambda^{2}$, it would have to be all of $G$. But this is not the case: setting

$$
Q=\frac{1}{2}\left(e_{1} \wedge e_{2}+e_{3} \wedge e_{4}+e_{2} \wedge e_{3}-e_{1} \wedge e_{4}\right)
$$

we have $Q \in G$ and $\sigma_{R}(Q)=1$.

Note that the $R$ of this example satisfies the first Bianchi identity, and also observe that this example illustrates the necessity of the assumption $\sigma_{R} \geq 0$ (or $\sigma_{R} \leq 0$ ) in Theorem 4.1.

Remark. Perhaps a word about the 3-dimensional case is in order at this point, even though it is included in the general case to be considered in the next section. For $n=3$, every 2 -vector is decomposable and hence $G$ is the entire unit sphere in $\Lambda^{2}$. Hence the critical values of $\sigma_{R}$ are just the eigenvalues of $R$, and the set of critical points of $\sigma_{R}$ with critical value $\lambda$ is just the intersection with $G$ of the $\lambda$-eigenspace of $R$. Note that this description (in dimension 3 ) is valid for each critical value $\lambda$, not just for the minimum and maximum values.

\section{The main theorem}

We now proceed to our main result by way of a sequence of rather technical lemmas.

Lemma 5.1. Let $R \in \mathscr{R}$ be such that $\sigma_{R} \geq 0$, and suppose $P, Q \in Z(R)$. Then there exists $S \in \mathscr{S}$ such that $\{P, Q\} \subset \operatorname{Ker}(R-S)$.

Proof. Choose an orthonormal basis $\left\{e_{1}, \cdots, e_{n}\right\}$ for $V$ such that $P=e_{1} \wedge e_{2}$ and $Q$ is contained in the span of $\left\{e_{1}, \cdots, e_{4}\right\}$, so that $Q=\sum_{i<j \leq 4} q_{i j} e_{i} \wedge e_{j}$ for some $q_{i j} \in R$. Since $Q$ is a critical point (a minimum) of $\sigma_{R}, R Q=\frac{1}{2}\left(\operatorname{grad} \sigma_{R}\right)(Q)$ is normal to $G$ at $Q$ so, by Lemma 3.1,

$$
R Q=\sum_{i<j<k<l} \nu_{i j k l} S_{i j k l}(Q)
$$

for some $\nu_{i j k l} \in \boldsymbol{R}$ (the component of $R Q$ in the direction of $Q$ is zero since $\langle R Q, Q\rangle=\sigma_{R}(Q)=0$ ). Note that the $\nu_{i j k l}$ are not uniquely determined since the $S_{i j k l}(Q)$ are not linearly independent.

Similarly (see the proof of Theorem 3.2),

$$
R P=\sum_{2<k<l} \mu_{12 k l} S_{12 k l}(P),
$$


where now the $\mu_{12 k l}$ are uniquely determined since the $S_{12 k l}(P)$ are orthonormal. Moreover, by the Remark following Corollary 3.3, $S_{1}=\Sigma \mu_{12 k l} S_{12 k l}$ is the unique operator in $\mathscr{A}(P)^{\perp}$ such that $P \in \operatorname{Ker}\left(R-S_{1}\right)$. Thus, by Theorem 2.1, it suffices to construct an $S_{2} \in \mathscr{S}$ such that $Q \in \operatorname{Ker}\left(R-S_{2}\right)$ and such that the orthogonal projection of $S_{2}$ onto $\mathscr{A}(P)^{\perp}$ is just $S_{1}$. But $\left\{S_{i j k l} \mid i<j<k<l\right\}$ is an orthogonal set in $\mathscr{S}, S_{12 k l} \in \mathscr{A}(P)^{\perp}$ for $2<k<l$, and $S_{i j k l} \in \mathscr{A}(P)$ for $(i, j) \neq(1,2)$, and so the orthogonal projection into $\mathscr{A}(P)^{\perp}$ of $\sum_{i<j<k<l} \nu_{i j k l} S_{i j k l}$ is just $\sum_{2<k<l} \nu_{12 k l} S_{12 k l}$. Thus we must show that we can choose $\tilde{\nu}_{i j k l} \in \boldsymbol{R}$ such that

$$
R Q=\sum_{i<j<k<l} \tilde{\nu}_{i j k l} S_{i j k l}(Q) \text { and } \tilde{\nu}_{12 k l}=\mu_{12 k l} \quad \text { for } 2<k<l .
$$

Step I. Given any $\nu_{i j k l}(i<j<k<l)$ such that (1) is satisfied, we shall show that $\nu_{1234}=\mu_{1234}$. Let $W=e_{1} \wedge e_{2} \wedge e_{3} \wedge e_{4} \in \Lambda^{4}$. Identifying $W$ with the oriented 4-dimensional subspace of $V$ spanned by $\left\{e_{1}, \cdots, e_{4}\right\}$ we have $P \subset W$ and $Q \subset W$, i.e., $P, Q \in \Lambda^{2}(W) \subset \Lambda^{2}(V)$. Letting $\pi_{W}: \Lambda^{2}(V) \rightarrow \Lambda^{2}(W)$ denote orthogonal projection, we have

$$
\begin{aligned}
\nu_{1234} & =\left\langle\nu_{1234} S_{1234}(Q), S_{1234}(Q)\right\rangle=\left\langle\pi_{W} \Sigma_{\nu_{i j k l}} S_{i j k l}(Q), S_{1234}(Q)\right\rangle \\
& =\left\langle\pi_{W} \circ R(Q), *_{W} Q\right\rangle,
\end{aligned}
$$

where $*_{W}$ is the star operator of $W$. Similarly,

$$
\mu_{1234}=\left\langle\pi_{W} \circ R(P), *_{W} P\right\rangle \text {. }
$$

But the restriction of $\pi_{W} \circ R$ to $\Lambda^{2}(W)$ is a curvature operator (with sectional curvature $\geq 0$ ) on the 4-dimensional space $W$, and $\{P, Q\}$ is contained in the zero set of this curvature operator. Hence, by Theorem 4.1 , there exists a unique $\mu \in R$ such that $P, Q \in \operatorname{Ker}\left(\pi_{W} \circ R-S^{\prime}\right)$ where $S^{\prime}=\mu *_{W}$. Thus

$$
\nu_{1234}=\left\langle\pi_{W} \circ R(Q), *_{W} Q\right\rangle=\left\langle\mu *_{W} Q, *_{W} Q\right\rangle=\mu,
$$

and similarly $\mu_{1234}=\mu$, so $\nu_{1234}=\mu_{1234}$.

Step II. We shall take advantage of the non-uniqueness of the remaining $\nu_{i j k l}$ in (1) to make essential alterations. In terms of the basis $\left\{e_{i} \wedge e_{j} \mid i<j\right\}$ for $\Lambda^{2}$, (1) becomes

$$
\begin{aligned}
& R Q=\nu_{1234} S_{1234}(Q)+\sum_{5 \leq k} {\left[\left(\nu_{123 k} q_{23}+\nu_{124 k} q_{24}+\nu_{134 k} q_{34}\right) e_{1} \wedge e_{k}\right.} \\
&+\left(-\nu_{123 k} q_{13}-\nu_{124 k} q_{14}+\nu_{234 k} q_{34}\right) e_{2} \wedge e_{k} \\
&+\left(\nu_{123 k} q_{12}-\nu_{134 k} q_{14}-\nu_{234 k} q_{24}\right) e_{3} \wedge e_{k} \\
&\left.+\left(\nu_{124 k} q_{12}+\nu_{134 k} q_{13}+\nu_{234 k} q_{23}\right) e_{4} \wedge e_{k}\right] \\
&+\sum_{5 \leq k<l}\left[\nu_{12 k l} q_{12}+\nu_{13 k l} q_{13}+\nu_{14 k l} q_{14}\right. \\
&\left.+\nu_{23 k l} q_{23}+\nu_{24 k l} q_{24}+\nu_{34 k l} q_{34}\right] e_{k} \wedge e_{l} .
\end{aligned}
$$


Case I. Assume $q_{34} \neq 0$. Then, given $\nu_{i j k l}$ satisfying (1), we can choose, for each $k \geq 5, \tilde{\nu}_{134 k}$ and $\tilde{\nu}_{234 k} \in \boldsymbol{R}$ so that

$$
\begin{gathered}
\mu_{123 k} q_{23}+\mu_{124 k} q_{24}+\tilde{\nu}_{134 k} q_{34}=\nu_{123 k} q_{23}+\nu_{124 k} q_{24}+\nu_{134 k} q_{34}, \\
-\tilde{\mu}_{123 k} q_{13}-\mu_{124 k} q_{14}+\tilde{\nu}_{234 k} q_{34}=-\nu_{123 k} q_{13}-\nu_{124 k} q_{14}+\nu_{234 k} q_{34} .
\end{gathered}
$$

(Compare (4) and (5) with the coefficients of $e_{1} \wedge e_{k}$ and $e_{2} \wedge e_{k}$ in (3).) Having chosen $\tilde{\nu}_{134 k}$ and $\tilde{\nu}_{234 k}$ to satisfy (4) and (5), note that

$$
\begin{aligned}
& \mu_{123 k} q_{12}-\tilde{\nu}_{134 k} q_{14}-\tilde{\nu}_{234 k} q_{24}=\nu_{123 k}\left(q_{13} q_{24}-q_{14} q_{23}\right) / q_{34} \\
& -\nu_{134 k} q_{14}-\nu_{234 k} q_{24}+\mu_{123 k}\left[q_{12}+\left(q_{14} q_{23}-q_{13} q_{24}\right) / q_{34}\right] .
\end{aligned}
$$

But

$$
q_{12} q_{34}+q_{14} q_{23}-q_{13} q_{24}=\frac{1}{2}\left\langle Q, *_{W} Q\right\rangle=0,
$$

so the above equation reduces to

$$
\mu_{123 k} q_{12}-\tilde{\nu}_{134 k} q_{14}-\tilde{\nu}_{234 k} q_{24}=\nu_{123 k} q_{12}-\nu_{134 k} q_{14}-\nu_{234 k} q_{24} .
$$

(Compare (6) with the coefficient of $e_{3} \wedge e_{k}$ in (3).)

Similarly we can check that

$$
\mu_{124 k} q_{12}+\tilde{\nu}_{134 k} q_{13}+\tilde{\nu}_{234 k} q_{23}=\nu_{124 k} q_{12}+\nu_{134 k} q_{13}+\nu_{234 k} q_{23} .
$$

(Compare (7) with the coefficient of $e_{4} \wedge e_{k}$ in (3).)

Finally, since $q_{34} \neq 0$ we can choose, for each $l>k \geq 5, \tilde{\nu}_{34 k l}$ such that

$$
\mu_{12 k l} q_{12}+\tilde{\nu}_{34 k l} q_{34}=\nu_{12 k l} q_{12}+\nu_{34 k l} q_{34} .
$$

(Compare (8) with the coefficient of $e_{k} \wedge e_{l}$ in (3).)

Then, setting $\tilde{\nu}_{12 k l}=\mu_{12 k l}$ for $2<k<l$ and $\tilde{\nu}_{i j k l}=\nu_{i j k l}$ for all $i, j, k, l$ for which $\tilde{\nu}_{i j k l}$ has not been previously defined, it follows from (1)-(8), together with step I, that

$$
R Q=\sum_{\nu_{i j k l}} S_{i j k l}(Q)=\Sigma_{\tilde{\nu}_{i j k l}} S_{i j k l}(Q),
$$

and $\tilde{\nu}_{12 k l}=\mu_{12 k l}$ for $2<k<l$. This completes the proof in the case where $q_{34} \neq 0$.

Case II. Suppose $q_{34}=0$. Then

$$
0=q_{34}=\left\langle Q, e_{3} \wedge e_{4}\right\rangle=\left\langle Q, *_{W} e_{1} \wedge e_{2}\right\rangle=\left\langle Q, *_{W} P\right\rangle=\langle P \wedge Q, W\rangle .
$$

But $P, Q \in \Lambda^{2}(W)$ implies $P \wedge Q$ is a multiple of $W$. Therefore $P \wedge Q=0$. It follows that the 2-planes $P$ and $Q$ have non-trivial intersection. Hence we can choose our basis $\left\{e_{1}, \cdots, e_{n}\right\}$ for $V$ so that $P=e_{1} \wedge e_{2}$ and 


$$
Q=e_{1} \wedge\left(q_{12} e_{2}+q_{13} e_{3}\right)=q_{12} e_{1} \wedge e_{2}+q_{13} e_{1} \wedge e_{3}
$$

for some $q_{12}, q_{13} \in \boldsymbol{R}$. Since $q_{14}=q_{23}=q_{24}=q_{34}=0$, (3) becomes

$$
\begin{gathered}
R Q=\nu_{1234} S_{1234}(Q)+\sum_{5 \leq k}\left[\nu_{123 k}\left(-q_{13} e_{2} \wedge e_{k}+q_{12} e_{3} \wedge e_{k}\right)\right. \\
\left.+\left(\nu_{124 k} q_{12}+\nu_{134 k} q_{13}\right) e_{4} \wedge e_{k}\right] \\
+\sum_{5 \leq k<l}\left(\nu_{12 k l} q_{12}+\nu_{13 k l} q_{13}\right) e_{k} \wedge e_{l} .
\end{gathered}
$$

Now $\nu_{1234}=\mu_{1234}$ since $P$ and $Q$ both lie in the 4-plane $e_{1} \wedge e_{2} \wedge e_{3} \wedge e_{4}$ (Step I). Similarly, $\nu_{123 k}=\mu_{123 k}$ for all $k \geq 4$ since $P$ and $Q$ both lie in the 4-plane $e_{1} \wedge e_{2} \wedge e_{3} \wedge e_{k}$. Moreover, $q_{13} \neq 0$ since $Q \neq P$, and hence we can choose $\tilde{\nu}_{134 k}(k \geq 5)$ and $\tilde{\nu}_{13 k l}(l>k \geq 5)$ such that

$$
\begin{aligned}
& \mu_{124 k} q_{12}+\tilde{\nu}_{13 k k} q_{13}=\nu_{124 k} q_{12}+\nu_{134 k} q_{13}, \\
& \mu_{12 k l} q_{12}+\tilde{\nu}_{13 k l} q_{13}=\nu_{12 k l} q_{12}+\nu_{13 k l} q_{13} .
\end{aligned}
$$

Then, setting $\tilde{\nu}_{12 k l}=\mu_{12 k l}$ for $2<k<l$ and $\tilde{\nu}_{i j k l}=\nu_{i j k l}$ for all $i, j, k, l$ for which $\tilde{\nu}_{i j k l}$ has not been previously defined, it follows from (1), $\left(3^{\prime}\right),\left(7^{\prime}\right)$ and $\left(8^{\prime}\right)$ that $R Q=\sum_{i j k l} S_{i j k l}(Q)$ and $\tilde{\nu}_{12 k l}=\mu_{12 k l}$ for $2<k<l$, as required.

Lemma 5.2. Let $Z \subset G$. Then there exists a finite subset $\left\{P_{1}, \cdots, P_{k}\right\}$ of $Z$ such that if $R \in \mathscr{R}$ and $P_{i} \in \operatorname{Ker}(R)$ for all $i \leq k$, then $Z \subset \operatorname{Ker} R$.

Proof. Suppose not. Then there exists an infinite sequence $\left\{P_{k}\right\}$ in $Z$ such that, for each $k, P_{k+1} \notin \operatorname{Ker}(R)$ for some $R \in \mathscr{R}$ with $\left\{P_{1}, \cdots, P_{k}\right\} \subset \operatorname{Ker}(R)$. But then

$$
\mathscr{R}_{k}=\left\{R \in \mathscr{R} \mid\left\{P_{1}, \cdots, P_{k}\right\} \subset \operatorname{Ker}(R)\right\}
$$

is a strictly decreasing infinite sequence of subspaces of $\mathscr{R}$, contradicting the finite dimensionality of $\mathscr{R}$.

Lemma 5.3. Let $X$ be an inner product space, and $X_{i}(1 \leq i \leq k)$ subspaces of $X$ such that $X=\sum_{i=1}^{k} X_{i}$. Let $\pi_{i}: X \rightarrow X_{i}$ and $\pi_{i j}: X \rightarrow X_{i} \cap X_{j}$ $(1 \leq i, j \leq k)$ denote orthogonal projections, and $x_{i} \in X_{i}(1 \leq i \leq k)$ be such that $\pi_{i j} x_{i}=\pi_{i j} x_{j}$ for all $i \neq j$. Then there exists a unique $x \in X$ such that $\pi_{i} x=x_{i}$ for all $i$.

Proof. An easy induction on $k$.

Theorem 5.4. Let $R \in \mathscr{R}$ be such that $\sigma_{R} \geq 0$. Then there exists $S \in \mathscr{S}$ such that $Z(R)=G \cap \operatorname{Ker}(R-S)$.

Proof. We shall construct the unique (see Theorem 2.2) $S \in \mathscr{A}(Z(R))^{\perp}$ which will do the job. By Lemma 5.2, there exists a finite subset $\left\{P_{1}, \cdots, P_{k}\right\}$ in $Z(R)$ such that every curvature operator which annihilates $\left\{P_{1}, \cdots, P_{k}\right\}$ annihilates $Z(R)$. In particular, 


$$
\mathscr{A}(Z(R))=\mathscr{A}\left(P_{1}, \cdots, P_{k}\right)=\bigcap_{1 \leq i \leq k} \mathscr{A}\left(P_{i}\right)
$$

and

$$
\mathscr{A}(Z(R))^{\perp}=\sum_{i=1}^{k} \mathscr{A}\left(P_{i}\right)^{\perp}
$$

For $i, j \leq k$, let $\pi_{i}: \mathscr{S} \rightarrow \mathscr{A}\left(P_{i}\right)^{\perp}$ and $\pi_{i j}: \mathscr{S} \rightarrow \mathscr{A}\left(P_{i}^{\perp}\right) \cap \mathscr{A}\left(P_{j}{ }^{\perp}\right)$ denote orthogonal projections. By Corollary 3.3, for each $i \leq k$ there exists $S_{i} \in \mathscr{A}\left(P_{i}\right)^{\perp}$ such that $P_{i} \in \operatorname{Ker}\left(R-S_{i}\right)$. Moreover, for $i \neq j, \pi_{i j}\left(S_{i}\right)=\pi_{i j}\left(S_{j}\right)$. Indeed, by Lemma 5.1, there exists $S_{i j} \in \mathscr{S}$ such that $\left\{P_{i}, P_{j}\right\} \subset \operatorname{Ker}\left(R-S_{i j}\right)$ and, by Theorem 2.1, $S_{i}=\pi_{i}\left(S_{i j}\right)$ and $S_{j}=\pi_{j}\left(S_{i j}\right)$ so $\pi_{i j}\left(S_{i}\right)=\pi_{i j}\left(S_{i j}\right)=\pi_{i j}\left(S_{j}\right)$. Hence, by Lemma 5.3, there exists $S \in \sum \mathscr{A}\left(P_{i}\right)^{\perp}=\mathscr{A}(Z(R))^{\perp}$ such that $\pi_{i}(S)$ $=S_{i}$ for all $i \leq k$. By Theorem 2.1 again, this implies that $P_{i} \in \operatorname{Ker}(R-S)$ for all $i \leq k$, and hence $Z(R) \subset \operatorname{Ker}(R-S)$ by the defining property of the set $\left\{P_{1}, \cdots, P_{k}\right\}$. Finally, $G \cap \operatorname{Ker}(R-S) \subset Z(R)$ by the remark in $\S 2$ and so we have the equality.

Corollary 5.5. Let $R \in \mathscr{R}$ and let $\lambda$ denote the minimum (or maximum) value of $\sigma_{R}$. Then there exists $S \in \mathscr{S}$ such that

$$
\left\{P \in G \mid \sigma_{R}(P)=\lambda\right\}=G \cap \operatorname{Ker}(R-\lambda I-S) .
$$

Proof. Immediate from Theorem 5.4 upon replacing $R$ in that theorem by $R-\lambda I$ (or, in the maximum case, by $\lambda I-R$ ).

Remarks. (i) It is interesting to note that the only use of the assumption that $\lambda$ be the minimum or maximum of $\sigma_{R}$ or, in Theorem 5.4, the assumption that $\sigma_{R} \geq 0$, occurs in the proof of the 4-dimensional case (Theorem 4.1). Thus, if it were true for 4-dimensional spaces that the set of critical points of $\sigma_{R}$ with critical value $\lambda$ were of the form $G \cap \operatorname{Ker}(R-S)$ for some $S \in \mathscr{S}$, then it would be true in general. Of course, it is not. The counterexample in $\S 4$ easily extends to all dimensions $\geq 4$.

(ii) Corollary 5.5 implies that there are linear subspaces $L_{1}$ and $L_{2}$ of $\Lambda^{2}$ such that $G \cap L_{1}$ is the minimum set of $\sigma_{R}$ and $G \cap L_{2}$ is the maximum set of $\sigma_{R}$. These subspaces can have non-trivial intersection. For example, let $\operatorname{dim} V=4$ and let $R \in \mathscr{R}$ be defined by

$$
\begin{aligned}
& R\left(e_{1} \wedge e_{2}\right)=R\left(e_{3} \wedge e_{4}\right)=e_{1} \wedge e_{2}+e_{3} \wedge e_{4}, \\
& R\left(e_{1} \wedge e_{3}\right)=R\left(e_{2} \wedge e_{4}\right)=0 \\
& R\left(e_{1} \wedge e_{4}\right)=R\left(e_{2} \wedge e_{3}\right)=-e_{1} \wedge e_{4}-e_{2} \wedge e_{3} .
\end{aligned}
$$

Then $L_{1}=\operatorname{Ker}(R+I+*), L_{2}=\operatorname{Ker}(R-I-*)$, and $\operatorname{dim}\left(L_{1} \cap L_{2}\right)=3$.

(iii) Given any linear subspace $L$ of $\Lambda^{2}$, there exists $R \in \mathscr{R}$ such that $\sigma_{R} \geq 0$ and $Z(R)=G \cap L$. Indeed, given $L$, the curvature operator $R$ which is zero on $L$ and identity on $L^{\perp}$ will have these properties. Moreover, the curvature 
operator obtained by projecting the one just described orthogonally onto $\mathscr{B}=\mathscr{S}^{\perp}$ will have these properties and will in addition satisfy the first Bianchi identity.

(iv) It is a consequence of Corollary 5.5 that if $M$ is an almost Kaehler manifold with almost complex structure $J$ and $m \in M$, then both the set of holomorphic 2-planes at $m$ (planes invariant under $J$ ) and the set of antiholomorphic 2-planes at $m$ (planes $P$ such that $v \in P$ implies $J v \perp P$ ) are intersections with $G$ of linear subspaces of $\Lambda^{2}(V)$ where $V=M_{m}$ is the tangent space of $M$ at $m$. Indeed, the automorphism $J$ of $V$ induces a curvature operator, also denoted by $J$, on $V$ by $J(u \wedge v)=J u \wedge J v(u, v \in V)$ and one easily checks that $\sigma_{J}$ assumes its maximum value 1 on holomorphic 2-planes and its minimum value 0 on anti-holomorphic 2-planes. A further computation verifies that in fact $P \in G$ is holomorphic if and only if $P \in \operatorname{Ker}(J-I)$, and $P \in G$ is anti-homomorphic if and only if $P \in \operatorname{Ker}(J-S)$ where $S \in \mathscr{S}$ is the operator corresponding under the isomorphisms of $\S 1$ to the 4-form $\varphi \wedge \varphi, \varphi$ being the fundamental 2-form given by $\varphi(u, v)=\langle J u, v\rangle$.

Added in proof. Theorem 5.4 has recently been generalized by $A$. Stehney to curvature operators on $\Lambda^{p}$ for arbitrary $p$. Using her techniques, it is possible to eliminate the intricate computations in the proof of Lemma 5.1.

\section{References}

[1] R. B. Gardner, Some applications of the retraction theorem in exterior algebra, J. Differential Geometry 2 (1968) 25-31.

[ 2 ] W. V. D. Hodge \& D. Pedoe, Methods of algebraic geometry, Cambridge University Press, Cambridge, 1947.

[3] I. M. Singer \& J. A. Thorpe, The curvature of 4-dimensional Einstein spaces, Global analysis, Papers in Honor of K. Kodaira, Princeton University Press, Princeton, 1969, 355-365.

[4] J. A. Thorpe, Curvature and the Petrov canonical forms, J. Mathematical Phys. 10 (1969) 1-7. 
